\section{LES POSTES A 120.000 VOLTS DE LA RÉGION PYRÉNÉENNE}

\section{Centrales d'Éget, de Loudenvielle et de Bordère}

Grâce au Ministère de l'armement et à la Compagnie des chemins de fer du Midi, la région Pyrénéenne possède, à l'heure actuelle el possédera cncorc, l'an prochain, leursínue le nouvcau programme en cours d'exécution sera réalisé, les lignes de transport d'énergie ì courants triphasés à la tension la plus élevée de celles exploilées en France.

Sur lc torrent de la Neste d'Aurc (Hautes-Pyrénées) est située l'usine d'Eget de la Compagnic des chemins de fer du Midi. Cette usine, située à r.or6 mètres d'altitude, a une chute de 750 mètres el une puissance utilisable de 35.000 HP.

Une deuxième usine siluée à Saint-Lary (fig. I) rescoil directement l'eau ì sa sortie de l'usine d'Eget.

L'usine d'Egel a fait l'objet d'études fort intéressinles de la part de M. Eydoux, Professcur supfléant à l'Ecole drs Ponts-et-Chaussées, à Paris et de M. Leboucher, Ingénicur en chef des Services techniques de la Compagnie des chrmins de fer du Midi (voir R. G. E. des ro et 3 r juillet I920), ainsi que de M. Barbillion, Directeur de l'Inslitut Electrotechnique de Grenoble (voir Industrie Electrique des ro aoûl et 25 septembre 1920). Nous n'avons rien à ajouter à ces études remarquables on tous points. Nous rappellcrons seulement les caractéristiques des machines de cette usine.

L'usine génératrice d'Eget possède :

Scpl allemateurs triphasés de la Général Electric Co (Thomson Américainc) de $3.500 \mathrm{kw}$. chacun, 6.000 volts 500 lours commandés par drs lubines Pellon construites par la Société Neyret Brylicr, de Grenoble.
Deux Dynamos d'excitation : l'une de $175 \mathrm{kw}$., l'autre de $60 \mathrm{kw}$, tension, $\mathrm{r} 25$ vols.

Six Transformaleurs-Elévaleurs 6.000/120.000 volts avec primaire connecté en triangle et secondaire en étoile aver

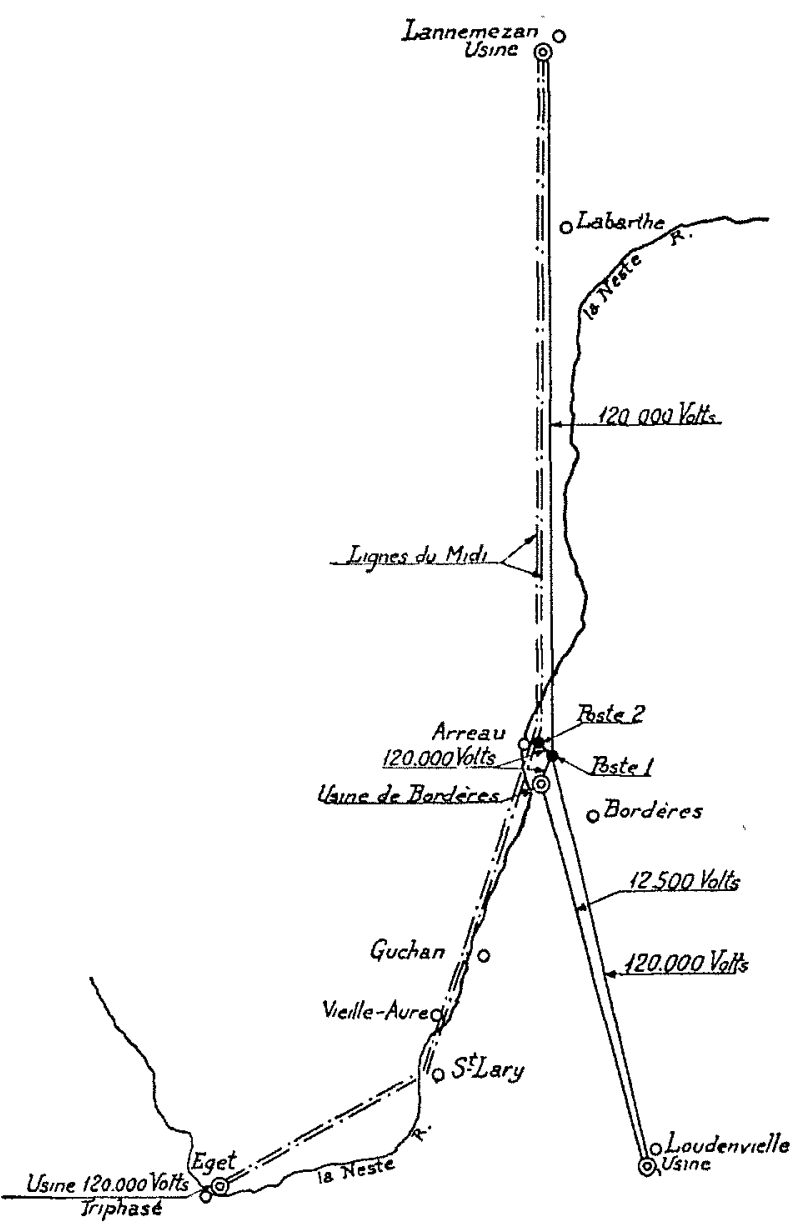

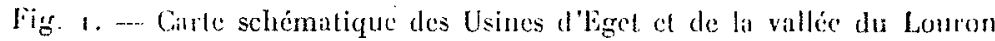

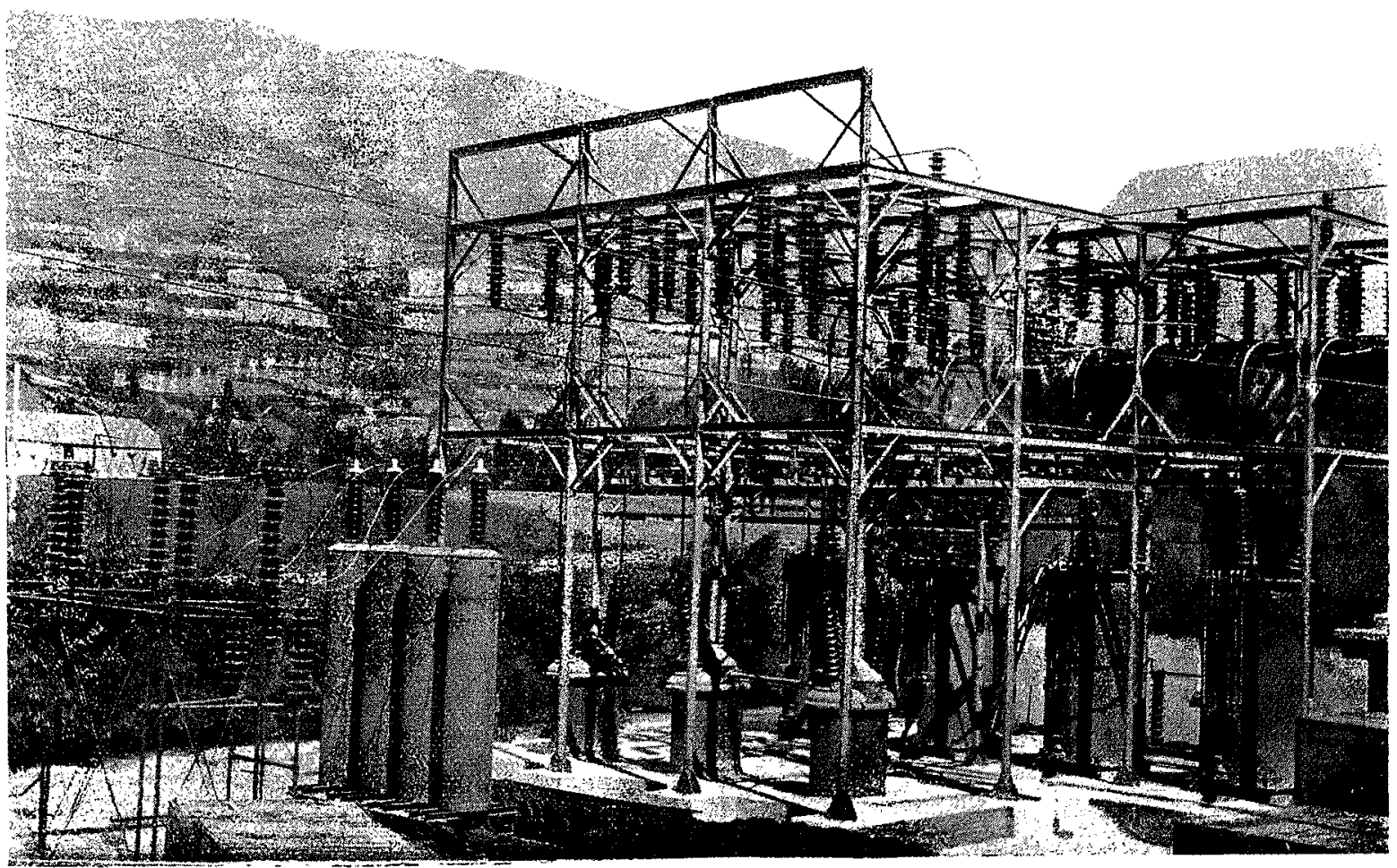

Fig. 2. -- Posle élćvaleur 6.000/5 20.000 volts, type « Outdoor » des Usines hydroẻlectriques de Loudenvielle et Bordères. 


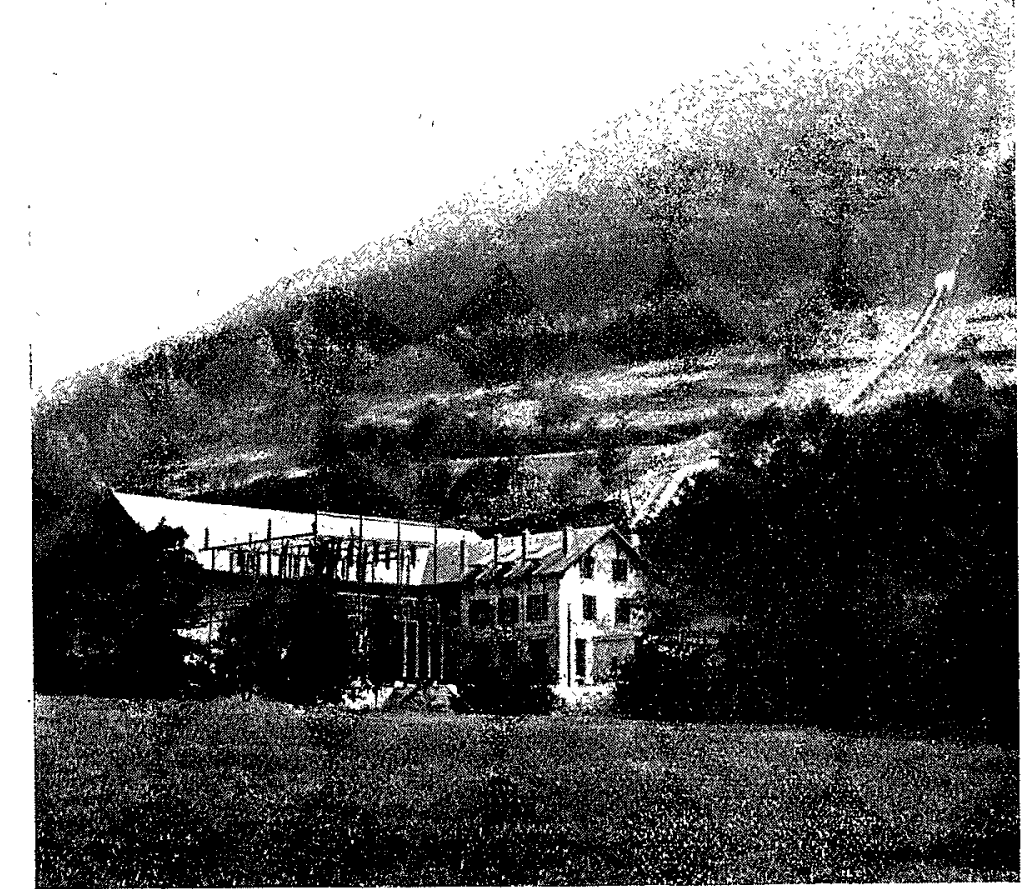

Fig. 3. - Vue d'ensemble de la centrale hydroćlectrique de Bordères. (En haut, on voit la conduite forcée ; en bas, all premier plan, lo poste élévalcur 120.000 volts en plein air). point neutre à la terre. Ces transformateurs débitent sıl deux lignes $\mathbf{2} 20.000$ volts pouvant être couplées à l'aide d'un interiupteur à huile.

Tout le matériel à r 20.000 volts se trouve à l'intéricur du bâtiment (Indoor Equipement) monté suivant la méthode ordinaire (apparcillage du type d'intérieur) ; scules, ls dimensions de l'appareillage, des distances 'entre fils, fils it masse attirent l'attention.

Toulcfois, la protection des lignes r 20.000 volts par des parafoudres électrolytiques se trouve placée à l'extérieur du bâtiment. La liaison de l'usine d'Eget avec les usines de Lolldenvielle et de Borderes a été prévuc.

Usines du Louron $\left(^{1}\right)$. - Comme nous venous de le voir, l'usine d'Eget possède un poste élévaleur 6.000/120.000 volls: du type d'intérieur. II n'en est pas de même des usines de Loudenviclle et de Bordères, de la valléc du Louron. Cos deux usines destinécs à alimenter les usines à carburc do Lannemezan ont été mises à exéculion pendant Ta gucrre parr lo Ministère de l'Armement, à une époque où il fallait fiarre extrèmement vite. Dos différents projels remis à colle époque, le Ministère de l'armement retint celui de la Société Westinghouse qui proposait un type de centrale très simple suivant la conception de la Westinghouse Elechical Compignie de Pittsburg avec poste ćlévalcur 120.000 volts du ly je en plein air (Ouldoor Equipenenl) (lig. 2). C'('st dans ces

(1) Voir R. G. E., 27/11/1920, Usines hydroelectriques du Lunpun (M. Warternaux).

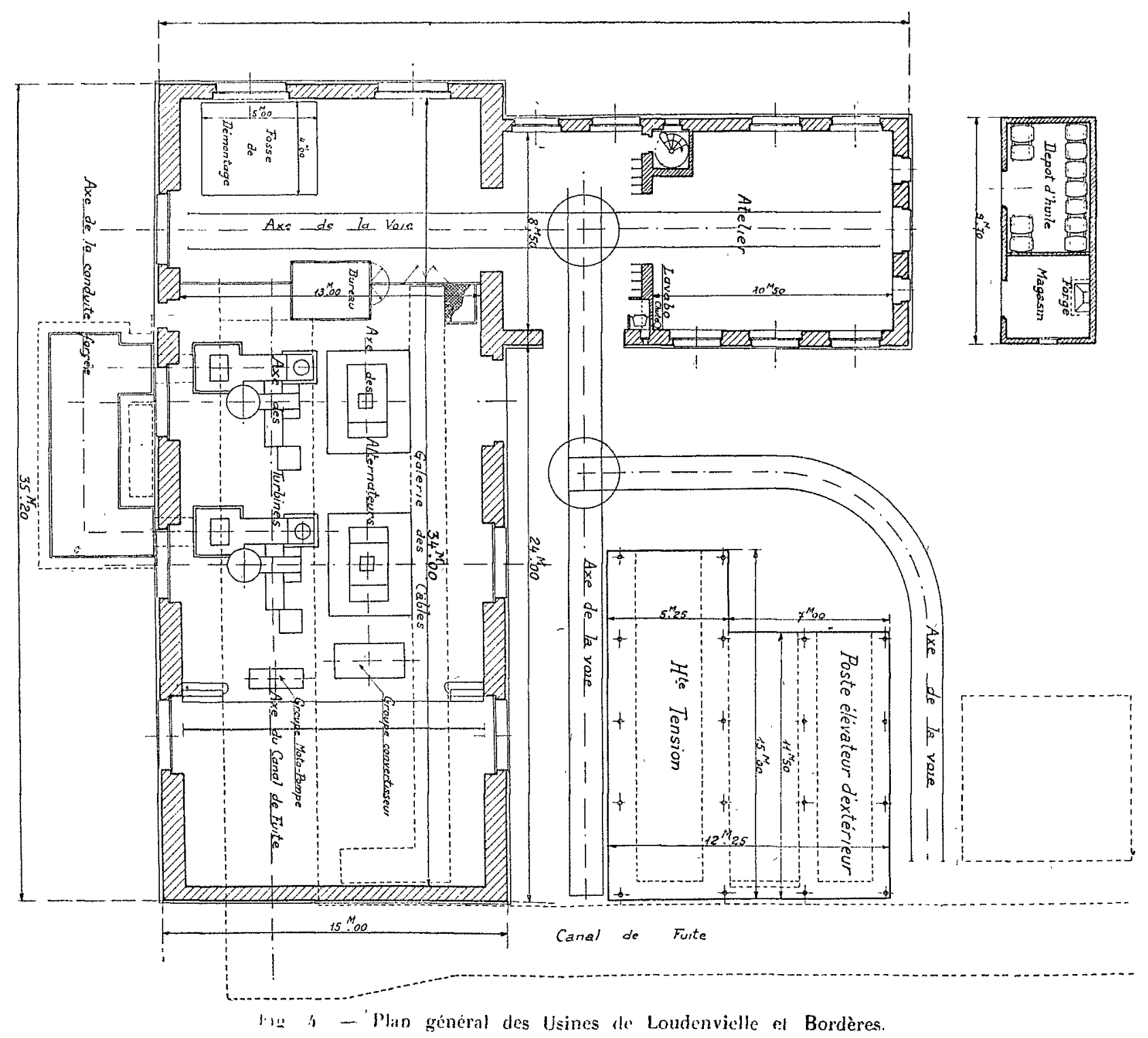




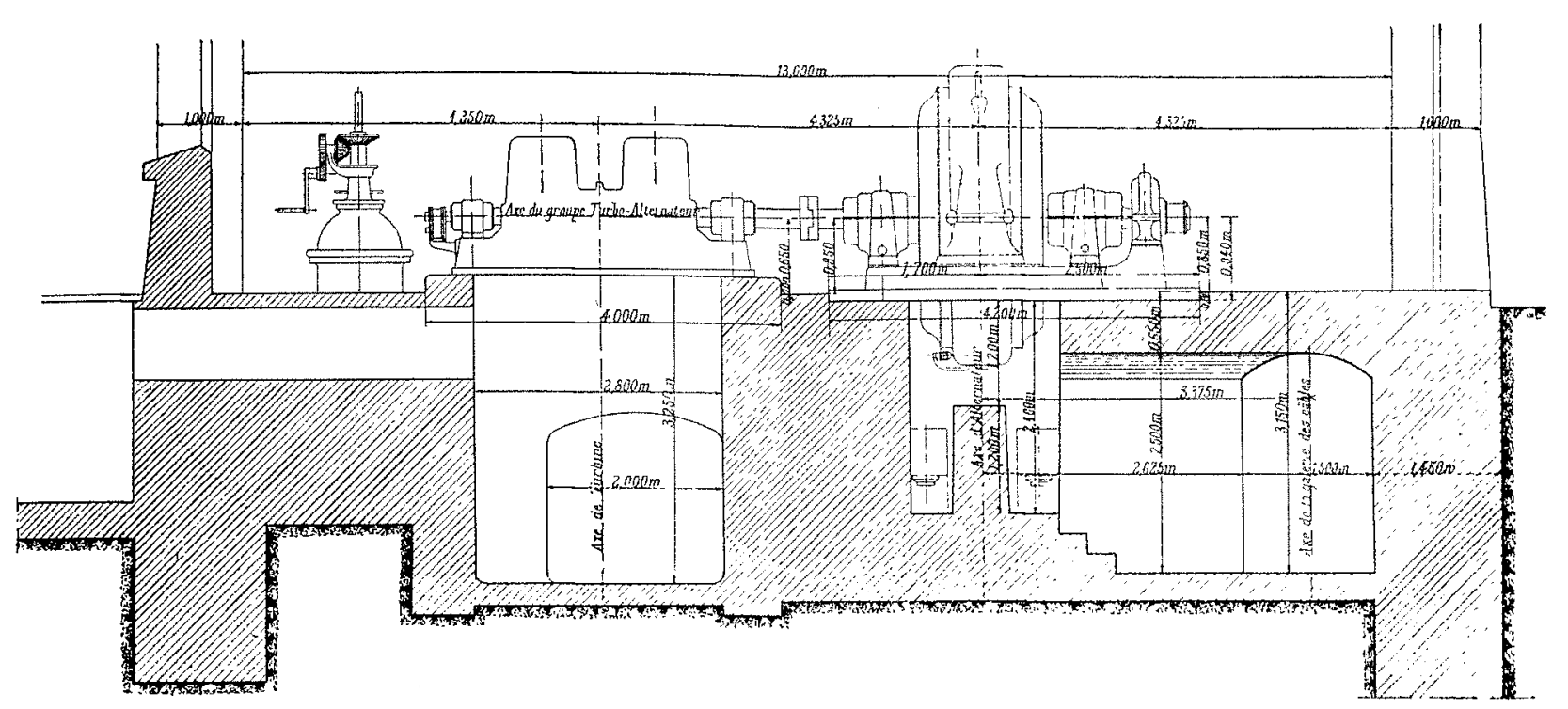

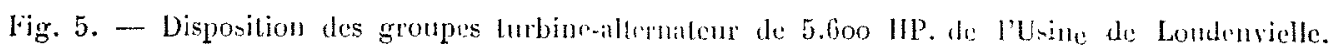

usines que, pour la première fois en France, on a adoplé des postes élévateurs du type extérieur "the Onldoor Equipement " des Américains. La Compagnic d'Electricité Industrielle a suivi le mouvement et a équipé de la mème façon les usines de luuchon el de Marignac.

Usine de Loudenvielle. - Cotle usine at situce sur li Neste du Louron, à proximilé du village de Loudenvielle.

L'eau est captéc à la cote $122 \mathrm{r}$, la chambre de mise en charge est à la cote 1216 . Lo débil varje de 1,0 ì h, I mèlres cubes à la seconde el la chule en caux moyennes est de 230 mètres.

Deux turbines Pelton de 5.600 HP à 5oo tours, onl élé foumies par la maison Leflaive el Cie, de Saint-Elicme.

Usine de Bordères (fig. 3). - Cetleu sine esl siluće en aval de celle de Loudenvielle. Sa chambre de mise en charge ( à la cole 835 mètres (sur la figr. 3 , on voil le postr éléraleri; en plein air).

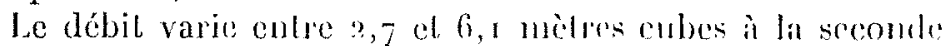
La chule effective on eaux mogemues ext de 165 mònes.

Deux lurbines de 5.600 He à 5oo lours onl égralement che fommics par la maison fellaive.

Au point de vue électrigue, les deus anines somb absolument identiques.

La figure 4 donne le plan génétal de l'usine hydro-éleclrique, la figure 5, la disposilion d'un groupe lumbine-allernateurs de 5.600 HP et la ligure 6 , une photographio de ce groupe.

I.e schéma général (fig. 7) s'explique de Jui-mêne :

Les deux allernateurs triphasés de 5.60o KVA, 6.000 volls, 50 périodes sont connectés sur un jeu de barres.

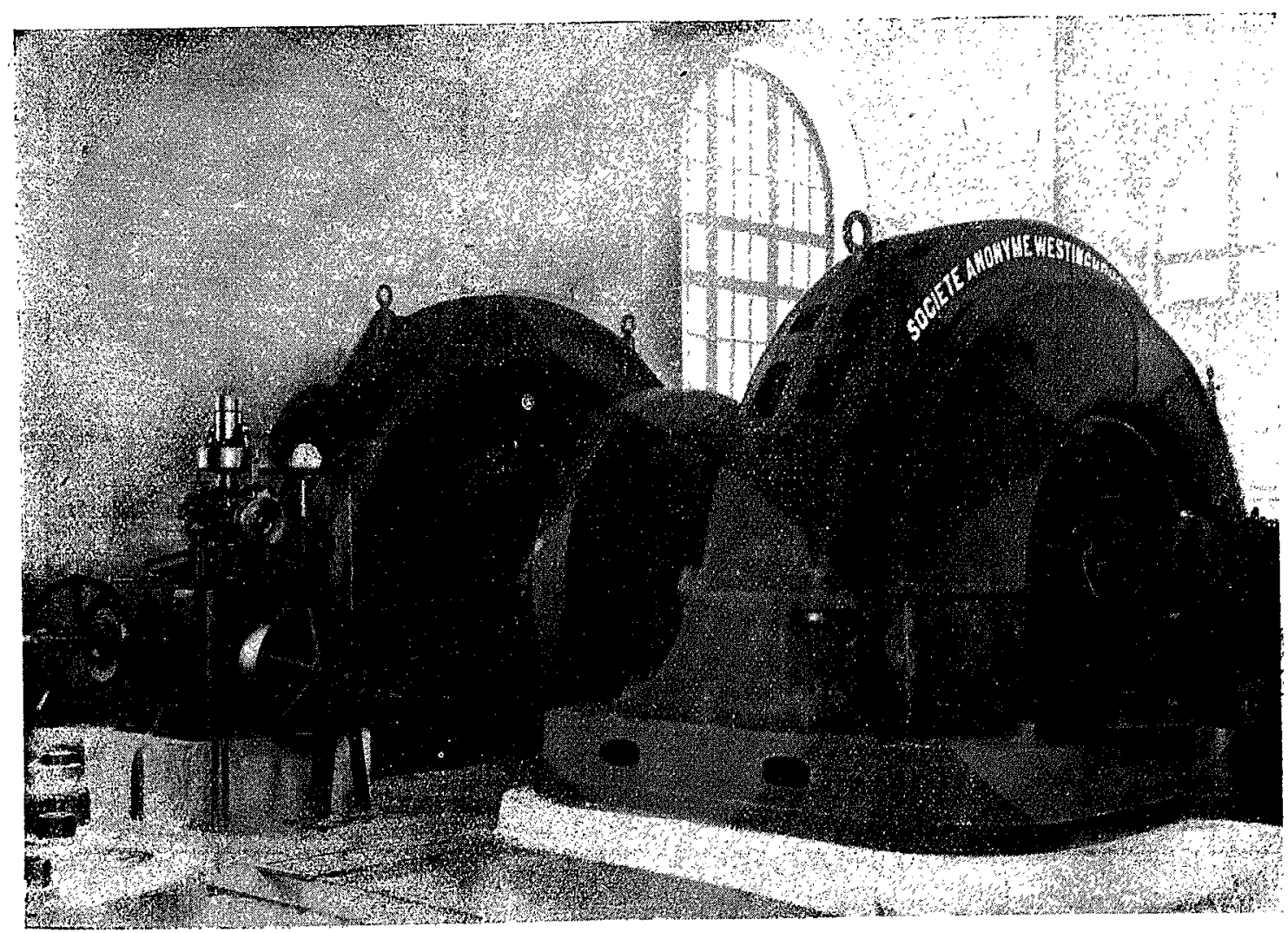

Fjog. 6. - Vue d'un groupe lurbine-alternateur de 5.600 HP., 500 tours, 6.000 volts, 50 périodes. 
Un seul départ 6.000 volts va vers le poste élévateur I 20.000 volts.

Deux départs secondaires à 6.000 volts sont prévus, l'un destiné à l'alimentation des services auxiliaires de la centrale comprenant un transformateur abaisseur de roo kw. rapport $6000 / 220$ volts, l'autre réscrvé à l'alimentation de villages de la vallée du Louron comprend un transformateur 6000/12000 volts de $300 \mathrm{KVA}$.
Alternaleur (fig. 6). - Les caractéristiques des altcrna teurs sont les suivantes :

Puissance : 5.600 KVA.

Tension $: 6.000$ volts.

Vitesse : 500 tours.

Fréquence : 50 périodes.

Poids de la partie tournante : environ 19,5 tonnes.

Poids total : 46,5 tonnes environ.

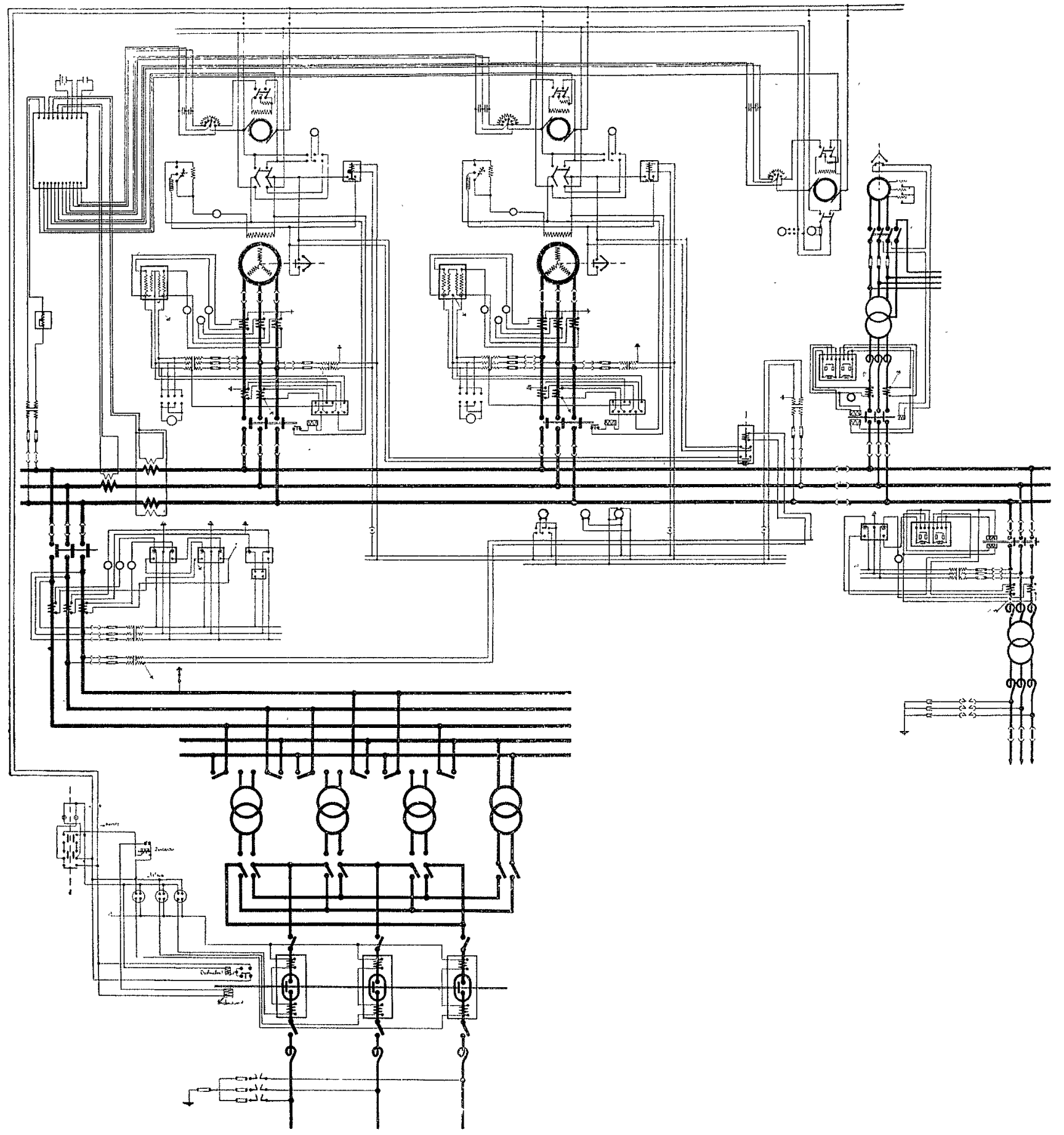

Fig. 7. - Schérna général des connexions des esintrales hydroćlectriques de Londunielle el de Borderes (conception Westinghouse).

De plus, les deux centrales sont reliécs par une ligne triphasée r 2.000 volts spéciale de secours.

Quant à l'cxcitation des alternateurs, elle est prévuc suivant la méthode couramment employée aujourd'hui dans les centrales modernes, par excitatrice directement montée en bout d'arbre de l'alternalcur. En outre, un groupe moteur triphasć, génératrice courant continu de $50 \mathrm{kw}$. peut servir de secours d'excitalion.
Chaque alternateur est accouplé direclement à sa turbine par manchon rigide.

Les paliers sont à refroidissement par circulation d'eau.

Etant donné la difficulté de transporter des pièces lourdes par les chemins de montage conduisant aux usines, on a été obligé de prévoir lo stator en deux pièces.

Les encoches du stator sont du type ouvertes. Il y en a cinq par pôle el par phase avec un bobinage à trois plans 


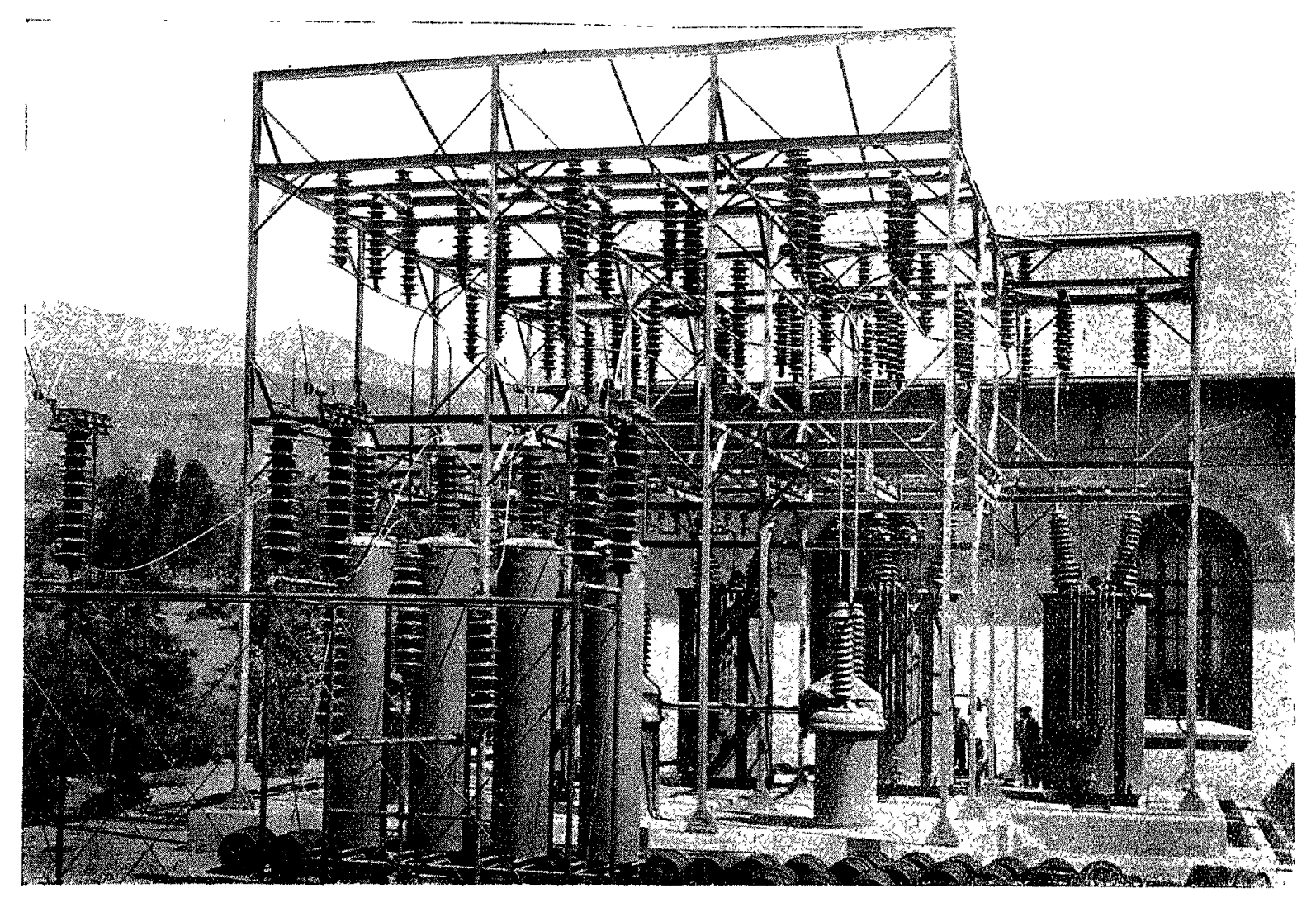

Fig. 8. - Poste élévateur 0.000/120.000 volts, type d'extérieur "Outdoor" des Usines du Louron.

permettant un démontage facile et rapide de la machine sans toucher aux enroulements.

Le rotor est constitué par des plaques d'acier assemblées par des boulons. Les pôles feuilletés sont assemblés sur le tambour à l'aide de queues d'aronde maintenues par clavellc

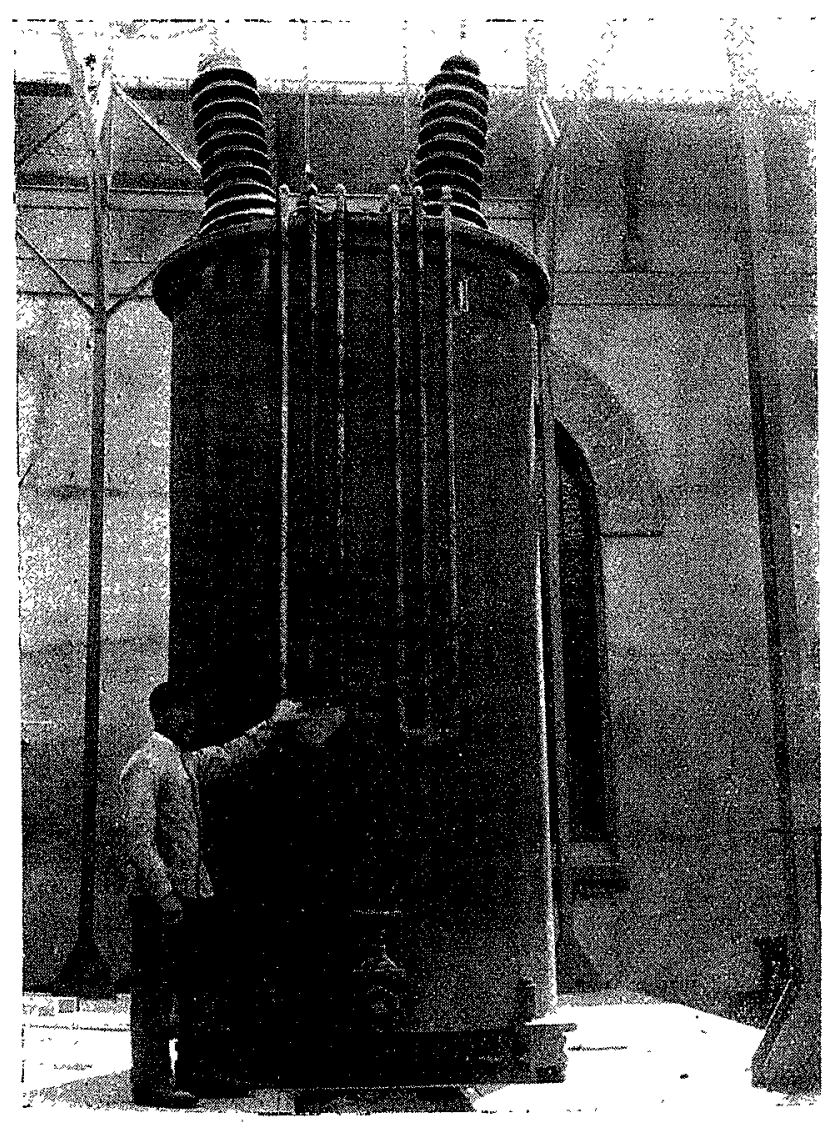

Fig. 9. - Transformateur monophasé dans l'huile, type Westinghous d'extéricur 4.000 KVA., 6.000/70.000 volts, 50 piriodes latérale. Ce dispositif résiste très bien aux grands efforts dus par les vitesses d'emballement de i, 8 fois la vitesse normale admise généralement dans les alternateurs commandés par turbine hydraulique.

Enfin, Ies pồles sont prévus avec circuits amortisscurs.

Appareillage colé 6.000 volls (voir schéma fig. 7).

Comme le montre le schéma général (fig. 7), l'apparcillage 6.000 volts est extrêmement simple.

On a prévu un seul jeu de barres 6.000 volts. Sur chaque alternateur, un disjoncteur à huile à déclanchement à maxima d'intensité à action différée et à retour d'énergic par relais type Weslinghouse, alimenté par les transformateurs d'intensité et de tension nécessaire. Un relais à surtension est, prévu sur chaçue alternateur ; son rôle est d'insérer automatiquement, lors d'une surtension de l'alternateur, une résistance supplémentaire dans le circuit d'excitation.

On remarque également (fig. 7) que chatgue allernaldur vossède un conlact à force centrifuge ruit, dans le cas d'emballement de l'alternateur, agit sur le relais à surtension ot tend à faire diminuer l'excitation. Sur les barres 6.000 voll: est prévu un relais à maximum de tension à circuit double qui est influencé par la tension de départ général.

Chaque alternateur poscède les apparcils de mesure néces saires au contrôle. : ampèremètres, vollmètres, watlmòlı indicateur.

Un waltmètre cnregistreur, un compleur, trois ampèremètres et un phasmètre sont branchés sur les barres 6.000 volts.

Les appareils de mise en phate des allernateurs, sont monfés sur bras mobiles el se composent d'un synchronoscop'. d'un fréquence-mètre à lames vibrantes, d'un voltmètre el de lampes.

Enfin, les alternateurs ont un régulaleur antomalique de tension du systeme Olmsted. Comme on te voil facilement sur le schéma de la figure ₹, ce régulateur agil sur l'excila- 
liọn des excilatrices par lintermédiaire d'une résistance lixe qui est mise en circuit ou hors circuit par le régulateur (principe du tout ou rien).

Poste élévaleur 6.000-70.000/120.000 volts (fig. 2 et 8).

Le courant produit à 6.000 volts par les alternaleurs de li centrale esl envoyé sur un jeu de barres aérien cl esl élevé, soil à 70.000 volts, soit à r 20.000 volts suivant le groupcment des enroulcments des transformateurs élévateur:, triangle ou étoile.

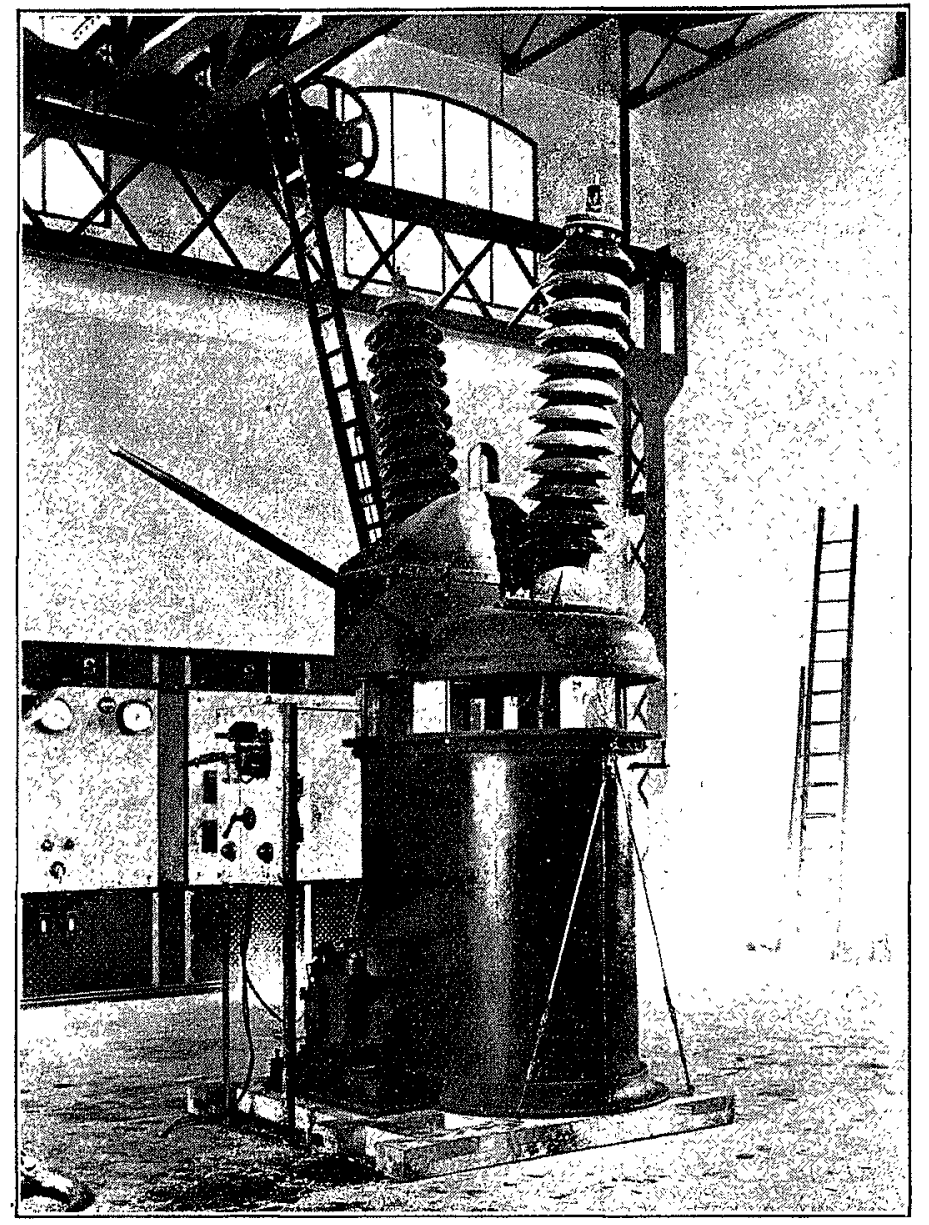

Fig. 10 - Pôle d'un disjoncteur 120060 rolts, type G A Westinghouse, en cours de montage en atelier. (On aperçoit, en haul el à gauche, le levier de manxutere it main, el en bas l'électro do commande à distance).

Ce poste 120.000 volts en plein air constitue, comme nous lavons dil, l'originalité de ces installations. Il est constitué simplement par une charpente métallique montée sur une plate-forme en béton. Celle-ci est prévue avec les canivcaux nécessaires au passage de la tuyauterie d'huile et d'eau et des commandes mécaniques.

Les $\Lambda$ méricains possèdent des lypes standard de postes "outdoor ", classés en catégorie de púissance et tension, el ont en stock les charpentes, les isolateurs en chapelet, les lransformaleurs, les disjoncteurs, elc..., de sorte que l'on peut commander un poste complet en donnant un simple numéro.

L'avanlage de ce genre de poste d'extérieur sur le type d'intérieur employé jusqu'à ce jour cn France, réside dans la grande économic réalisée sur le bâtiment, la rapidité de lcur montage, leur clarté permettant d'un scul coup d'œil de voir loute l'installation.

La charpente des postes de Loudenviclle el de Borgères (fig. 2 et 8) pèse environ 16 tonnes.
Transformateurs Elévatcurs 6000-70.000/120.000 volls. Ces transformateurs (fig. 9) sont du type Westinghous! monophasés dans l'huile à refroidissement par circulation d'eau.

Ils sont prévus égalemenl pour montage à l'extérieur.

Jeurs caractérisliques sont les suivanles :

Puissance unitaire: $4.000 \mathrm{KVA}$.

Fréquence : 50 périodes.

Ghaque poste élévaleur est conslitué par quatre transformalcurs idenliques, dont lrois sont en service ct un en réserve.

Lorsque les cnroulements haule tension sont monlés en triangle, le rapport de transformation est de 6.000/70.000 volts ef lorsque le montage esl en étoile $6.000 / 120.000$ volts.

Toules les manoures de branchement se font it litide de sectionneurs (voir schéma fig. 7).

On aperçoit sur la figure 9 , une vanne permeltant lér. cuation rapide de l'huile. Il cxiste aussi des prises pour to filtrage el le remplissage. Chaque transformaleur est mui

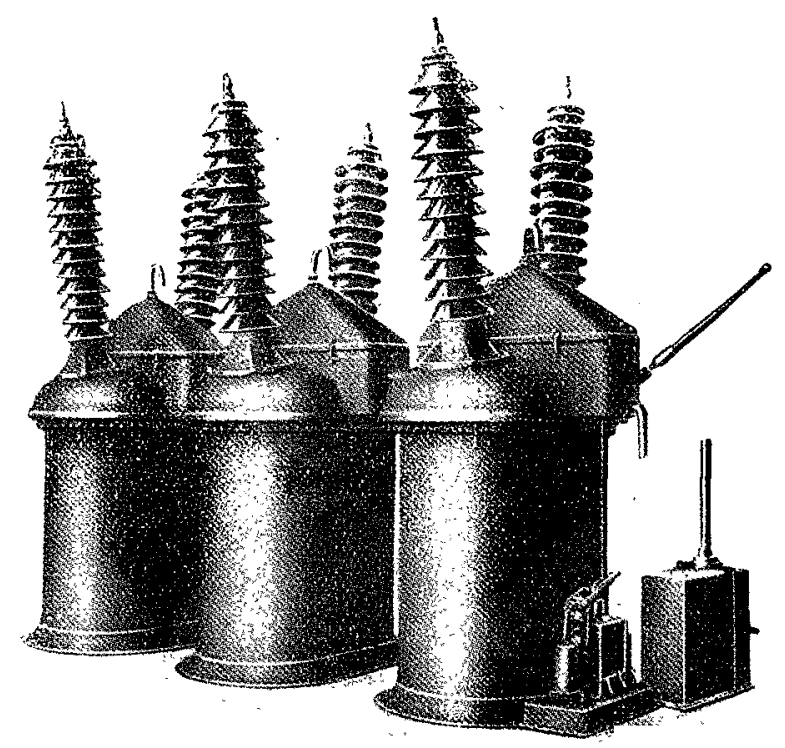

Fig. 11. - Disjoncleur tripolaire dans l'huile, type 120.000 volts d'extéricur, à commande à main par levier et électrique à distance - Capacité de rupture. $325.000 \mathrm{KVA}$.

d'un niveau d'huilc, d'un indicateur de température à con lact réglable el d'un diaphragme ayant pour but d'éviler ul ćclatement de la cuve au cas où une explosion se produirail à l'intérieur du transformatcur.

I'n point intéressant à signaler est que les bornes 120.010 volts sont du type condensateur, c'est-à-dire qu'clles ont ét éludiées spécialement de façon à répartir le plus uniformé ment possible, le champ ólcctro-slatique existant entre lo conducteur central el la masse.

Ces bornes sont scellées sur le couvercle du transformit teur à l'aide d'un ciment spécial et de joints cumpecchant toule introduction d'humidité dans l'appareil. Ces joints ont fail leur preuve dans des installations en Amérique du Nord où la pluié est abondante ct où les hivers sont très rigoureux ; les transformateurs marchent très bien sous d'épaisses souches de neige.

Les enroulements de ces transformatcurs ont unc grandr rigidité parce que leurs noyaux sont du type cuirassé, c'està-dire que le circuit magnélique enveloppe les enroulrments.

Celle grande rigidité leur permet de supporter, sans qu'il 
en résulte une déformation des cnroulements, les courtscircuits les plus violents du côté haute tensiun.

La figure 9 permet de se rendre compte des dimensions considérables de ce transformateur. Son poids avec huile est d'environ 2 I lónnes. Le poids d'huile élant de 7 tonnes environ.

Les pertes à vide sont de 30.000 watts environ, la réaclance 9 pour roo environ.

Les surcharges garanties par le cahier des charges sont:

25 pour roo pendant 2 heures.

roo pour roo momentanément, sans que l'élévation de tempéralure soit dangereuse. ruplure se produisant cependant dans une zone de la chre ai l'huile a encore son entière purcté.

Les contacts mobites sont éludićs de telle sorte qu'ils s'appliquent simultanément sur les contarls fives, avec mme pression égale délerminće par des ressorls. Dess shumls pretvus entre les conlacts mobiles évitent que le passage du courant n'endommage les ressorts de compression. La course d'ouverture est grande, environ $1.200 \mathrm{~mm}$.

La rupture esl double par póle et la lige qui supporte les conlacls mobiles établit en quralque sorte par sa forme 111 cloisonnement entre les deux points de ruplure, ce qui donne dans la pratique, un bou fonctionnement pour les très haules lensions supérinurs à 100.000 volls.

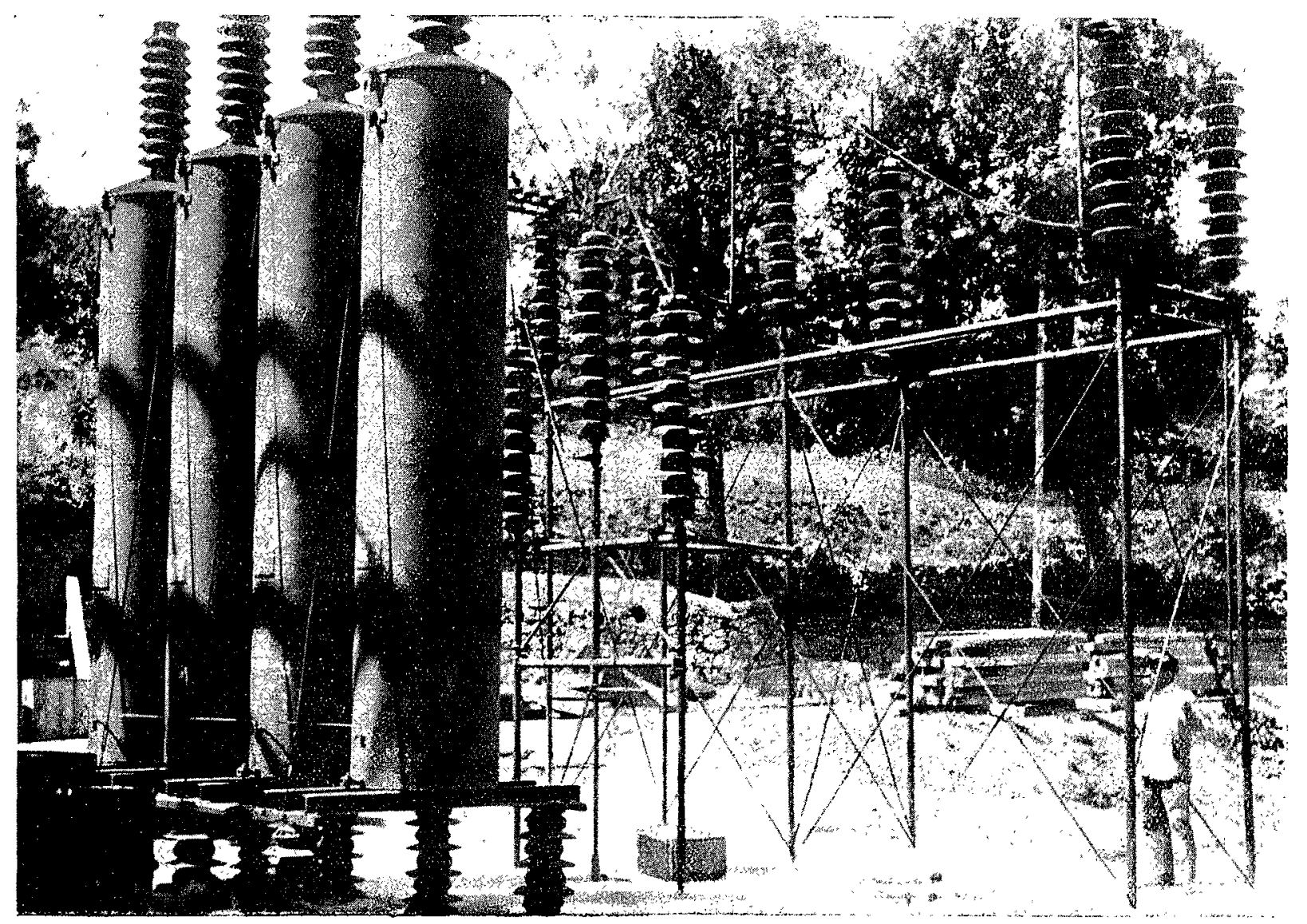

Fig. I2. - Parafoudre électrolytique Westinghouse, 120.000 volts, du lype « Unteloor ".

\section{Disjoncteur $\mathbf{2} 20.000$ volts.}

Comme on le voit sur le schéma général (figure 7), il n'existe qu'un seul départ i20.000 volts. Sur ce départ es! monté un disjoncteur tripolaire dans l'huile à commande ì main el automalique à distance.

Ce disjoncteur est du type G.A. "Weslinghouse " pour monlage à l'extérieur. I a figure to représente un pôle de ce lype de disjoncleur 120.000 volls en cours de monlage à l'usine du Bourget de la Compagnie Electro-Mécanique. Gr. lype de disjoncteur consiste (fig. I I, voir aussi fig. 2 et 8) en trois pôles enfermés chacun dans une cuve d'acier très robuste avec chemise isolante intérieure. Un couvercle de fonte couvre la cuve et porte le mécanisme de commande propre à chaque pôle. La commande simullanée des différents pòles se fail par tiges rigides actionnées soit par élecIro, soit par un levier à main. Chaque pôle peut êtrc ajusté séparément, ce qui rend très facile le réglage el l'entreticn.

Les contacts fixes sont fixés aux isolateurs qui supportent la borne de sortie. Les contacts sont plongés dans l'huile à une profondeur qui assure le soufflage efficace des arcs, la
Les bornes sont du lype condensalour. Ce lype de borne permet de diminuer le diamètre des parties isolanles ol, de ce fail, l'encombremenl de l'apparcil.

De plus, ces bornes sont munies à l'intéricur dr: la cuve du disjoncteur de "Bushings " formant transformaleurs d'intensité permetant d'alimenter les relais à maximum rel a action différée.

Etant donné le faible diamètre de la borne, l'exactitude de ces transformaleurs "Bushings i est supérieure à celle des transformalcurs monlés sur porcelinine. Ces bornes condensaleurs portent, en outre, à l'intéricur des assietles en porceiaine scellées au moyen d'un ciment spécial enpêchant toule introduction d'humidité dans la borne proprement dite. Cus porcelaines prolègent la partie active de celle borne contre la pluie et la neige.

I.e disjoncteur tripolaire r20.000 volts (fig. 7) des postrs de Loudenvielle el de Borderes est muni d'un disposilif d'ouverture et de fermelure à distanee actionne par courant continu 8o/roo volts. Ia commande dectrique ì distance so fait depuis la contrale. Le comant nécessaire à l'enclanchr- 


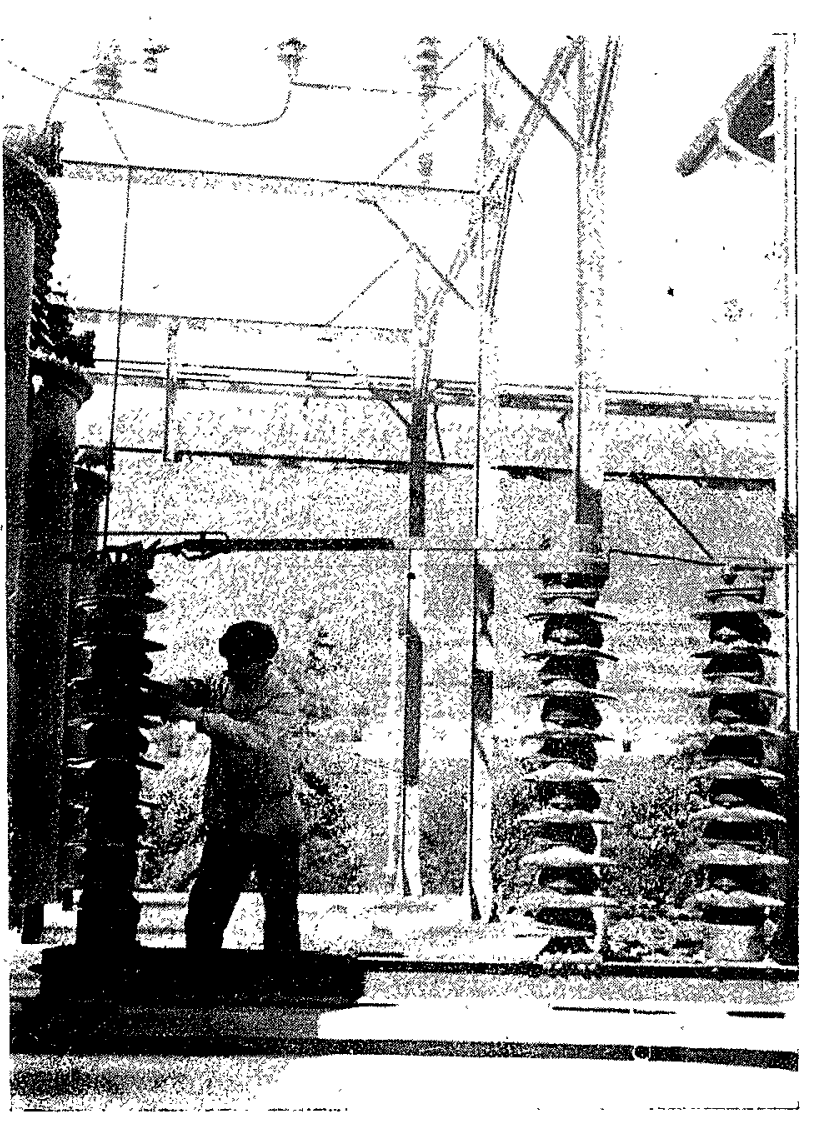

Fig. I3. - Sectionncur unipolaire 120.000 volts, pour poste de coupurc en plein air. d'effectuer les manouvres d'enclanchement et de déclanche. ment du disjoncleur à I 20,000 volts;

\section{Deux lampes témoin ;}

Un volant de manœuvre pour le déclanchement mécanique à distance du disjoncleur ;

Trois relais lype Westinghousc à maxima el à action différée.

I.es connexions entre lc petit panncau en question ot le lisjonctcur lui-même sont faites en câble armé.

la protection de la ligne r:0.000 volts est faite unique meǹt par une batterie de parafoudres électrolytiques, type $A R$ avec point neutre isolé. Le principe de ces apparuils cst bien connu, il consiste en des séries d'assiettes en aluminium empilées les unes sur les autres et plongées dans un ćlectrolyte approprić. En temps normal, l'écoulement du courant de ligne est limité par une couche d'hydroxycle d'alumine, mais dès qu'une surtension se produil, celte couche est percée et le courant s'écoule à la terre. Des polites cornes sont montées en série avec le parafoudre éleclrolylique afin de limiter le courant de terre. De temps en temps, il cst néccssaire de reformer la couche d'hydroxyde en amorçant le courant de terre, ce qui produit parfois des étincelles d'un effet curieux.

La figure i2 représente un tel parafoudre électrolytique r20.000 volts en plein air. Ces appareils sont nettement visibles sur les figures 2 et 8 .

Les connexions du poste élévateur ne sont pas on fil, mais sont en tubes de fer étiré de 49 millimètres de diannètre.

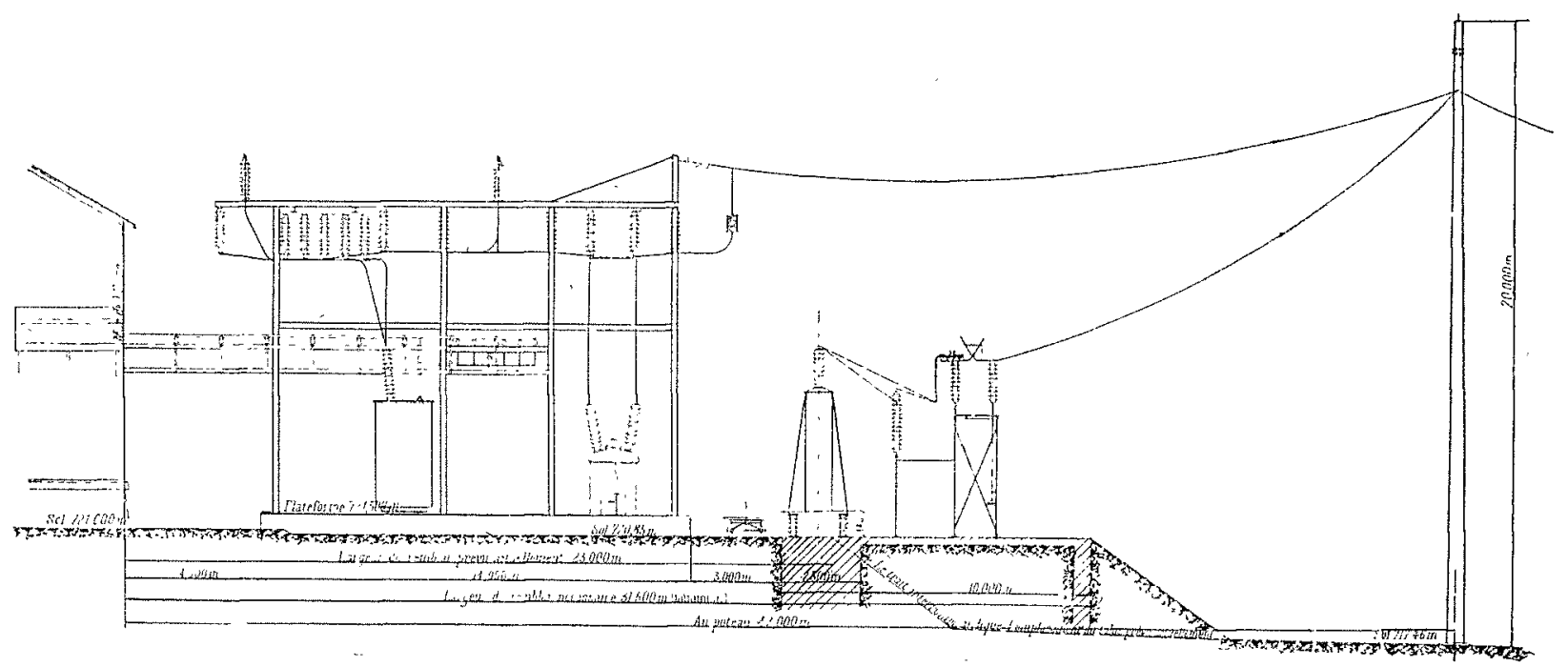

Fïg. I/. - Installialion des parafoulders électrolytiques et sortic de ligne, r mo.0oo volls.

ment est de roo ampieres environ. Il existe igalement un dispositif de commande mécanique par câble permoltant le déclanchement depuis la centrale. Enfin, le rérnclanchement peut se faire à la main par le Ievier qui est placé sur lo disjoncteur lui-même.

Les conlacts du disjoncleur sont prévus pour 300 ampères, ct sa capacité de rupture est de $325.000 \mathrm{KVA}$, valeur relativement considérable. I a hauteur totale est d'environ $3 \mathrm{~m} .65$ .l la contenance d'huile par pôle environ r.5on kgs.

I.es apparcils basse tension de contròle du disjoncteur sont placés à l'intéricur de la centrale montés sur un petit panneau portant :

Lin controller combiné avec un contacteur el permettanl
Les isolatenrs supports de lubes, de même que ceux des sechionncurs (fig. I3), sonl constilués par neuf assiclles en porcelaine empilées les unes sur les autres. Ges assiettes sont loules du même modèle of sont nunies d'une cmbase ch dune calotte en fonte ct pearvent servir soil comme isolalcur à chapelel suspendu, so:l commo isolateur support ardinaire.

Enfin, la figure i 4 représente le départ de la ligne 20.000 volts faisant la jonction cntre Jes centrales du Louron th l'usine réccptrice de Lannemezan. Celle ligne esl en câble de cuivre de roo $\mathrm{mm}^{2}$ de scetion.

V. Sytuestre,

Ingénieur A. M. el $I . E$. G. 